\title{
LA INTERVENCIÓN DE LA UE FRENTE A LAS CONSECUENCIAS ECONÓMICAS DE LA PANDEMIA DE COVID-19 Y SU ARTICULACIÓN JURÍDICA
}

\author{
Alberto Macho Carro \\ Investigador predoctoral en el área de Derecho Constitucional \\ Universidad de Valladolid
}

Cómo citar este artículo / Citation: Macho Carro, A. (2020). La intervención de la UE frente a las consecuencias económicas de la pandemia de Covid-19 y su articulación jurídica. Biglino Campos, P.; Durán Alba, F. Los Efectos Horizontales de la COVID sobre el sistema constitucional, Colección Obras colectivas, Fundación Manuel Giménez Abad, Zaragoza. DOI: https://doi.org/10.47919/FMGA.OC20.0018

SUMARIO: I. INTRODUCCIÓN - II. LA FLEXIBILIZACIÓN DE LA NORMATIVA EUROPEA PARA FACILITAR LA RESPUESTA ESTATAL A LAS CONSECUENCIAS ECONÓMICAS DE LA PANDEMIA - 1. La flexibilización de la normativa europea sobre ayudas de Estado - 2. La flexibilización del marco presupuestario europeo - III. LA INTERVENCIÓN DEL BANCO CENTRAL EUROPEO. IV. LOS MECANISMOS FINANCIEROS DE LA UNIÓN EUROPEA PARA COMBATIR LAS CONSECUENCIAS ECONÓMICAS DE LA PANDEMIA - 1. El instrumento europeo de apoyo temporal para mitigar los riesgos de desempleo en una emergencia - 2. Medidas para facilitar la liquidez a través del Grupo del Banco Europeo de Inversiones - 3. De la apuesta fallida por los "coronabonos" a la eliminación de la condicionalidad del MEDE - V. LA GESTACIÓN DEL PLAN DE RECUPERACIÓN EUROPEO FRENTE A LA PANDEMIA: NEXT GENERATION EU - 1. La propuesta de la Comisión Europea - 2. La negociación del plan de recuperación en el Consejo Europeo VI. CONCLUSIONES. 


\section{INTRODUCCIÓN}

Como es sabido, la pandemia de covid-19 originada en China a finales de 2019 golpeó Europa de forma especialmente intensa en los primeros meses de 2020. Este impacto, si bien profundamente desigual en términos de contagio y mortalidad, obligó a prácticamente todos los países de la Unión Europea a decretar medidas excepcionales para restringir la movilidad de sus ciudadanos y reducir así la propagación del virus. Unas medidas que en muchos casos se extendieron durante meses y que inmediatamente se tradujeron en un brusco parón de toda actividad económica no esencial.

Con la intención de paliar en la medida de lo posible la magnitud de una crisis económica inevitable, los Estados miembros de la UE, primero, y la propia UE, después, comenzaron a adoptar decisiones orientadas a propiciar la pervivencia de las empresas y puestos de trabajo, así como a impulsar la economía una vez levantadas las medidas de confinamiento.

El presente trabajo pretende dar cuenta de cómo se ha articulado jurídicamente la respuesta de la Unión Europea a las consecuencias económicas ocasionadas por la pandemia de covid-19. En este sentido, se analiza en primer lugar la flexibilización operada en el marco normativo comunitario sobre ayudas de Estado y estabilidad presupuestaria para amparar diversas medidas económicas de los Estados miembros. A continuación, se refiere cuál ha sido el papel del Banco Central Europeo para respaldar esta respuesta. En tercer lugar, se centra el foco en las medidas financieras más relevantes adoptadas por la propia UE para complementar la actuación de los Estados: tres "redes de seguridad" que han movilizado 540.000 millones de euros. Finalmente, se desgrana el proceso de gestación y negociación de un fondo de recuperación sin precedentes en la historia de la UE: el Next Generation EU. 
II. LA FLEXIBILIZACIÓN DE LA NORMATIVA EUROPEA PARA FACILITAR LA RESPUESTA ESTATAL A LAS CONSECUENCIAS ECONÓMICAS DE LA PANDEMIA

1. La flexibilización de la normativa europea sobre ayudas de Estado

Uno de los primeros ámbitos donde la Comisión Europea intervino para facilitar una rápida respuesta a los daños económicos ocasionados por la pandemia de covid-19 ha sido el de las llamadas ayudas de Estado.

En principio, de acuerdo con lo establecido en el artículo 107.1 TFUE, "salvo que los Tratados dispongan otra cosa, serán incompatibles con el mercado interior, en la medida en que afecten a los intercambios comerciales entre Estados miembros, las ayudas otorgadas por los Estados o mediante fondos estatales, bajo cualquier forma, que falseen 0 amenacen falsear la competencia, favoreciendo a determinadas empresas o producciones". Sin embargo, los apartados subsiguientes de este precepto establecen una serie de excepciones a esa incompatibilidad, y la Comisión no tardó en recurrir a ellas para facilitar que los Estados miembros pudieran acudir al rescate de sus empresas, que en muchos casos sufrieron graves problemas de liquidez a causa de la falta de ingresos ocasionada por la pandemia. Así, mediante una comunicación de 13 de marzo sobre la "respuesta económica coordinada al brote de covid-19"1, la Comisión abrió la puerta a las siguientes medidas de apoyo.

En primer lugar, la posibilidad de que los Estados miembros adopten medidas que no entran en el ámbito del control de las ayudas estatales y que, por tanto, pueden ser implementadas inmediatamente, sin contar con la Comisión. Dentro de esta categoría destacan, por un lado, algunas medidas destinadas a aliviar la presión financiera sobre las empresas, como por ejemplo los subsidios salariales y la suspensión del pago del impuesto de sociedades y del IVA o de las cotizaciones sociales. Por otro lado, medidas de apoyo financiero directo a los consumidores, como en casos de servicios o billetes cancelados que no hayan sido reembolsados por los operadores correspondientes.

\footnotetext{
${ }^{1}$ Comisión Europea, "Respuesta económica coordinada al brote de COVID-19", COM (2020) 112 final, de 13 de marzo.
} 
En segundo lugar, se contempla la posibilidad, de acuerdo con el artículo 107.3.c), de autorizar "ayudas destinadas a facilitar el desarrollo de determinadas actividades", lo que — según la propia comunicación— se traduce en la posibilidad de que los Estados miembros, previa aprobación por la Comisión, puedan "responder a las necesidades agudas de liquidez y apoyar a empresas amenazadas de quiebra por el brote de covid-19".

En tercer lugar, se prevé también la posibilidad de aprobar "ayudas destinadas a reparar los perjuicios causados por desastres naturales o por otros acontecimientos de carácter excepcional", consideradas compatibles con el mercado interior por el artículo 107.2.b) TFUE. Esta disposición permite a los Estados miembros, previa aprobación por la Comisión, compensar a las empresas por el daño sufrido en circunstancias excepcionales, como sin duda lo son las causadas por el brote de covid-19. Aquí se incluyen medidas para resarcir a las empresas de aquellos sectores que se han visto especialmente afectados por la pandemia, como por ejemplo el transporte, el turismo o la hostelería; pero también ayudas para compensar a los organizadores de actos cancelados por los daños ocasionados.

Interesa destacar que la noción de "acontecimientos de carácter excepcional" contemplada en el artículo 107.2.b) ha venido siendo interpretada de forma muy restrictiva por la Comisión, hasta el punto de que esta norma solo se había invocado con éxito en contadas ocasiones, como por ejemplo en el caso de la propagación de la denominada "enfermedad de las vacas locas" (Santa María, Gambaro, Missanelli, 2020).

Por último -aunque quizá lo más importante-, ya en esta comunicación la Comisión consideró que la naturaleza y magnitud del impacto que la pandemia estaba teniendo en Italia permitía recurrir al artículo 107.3.b) TFUE, según el cual "podrán" considerarse compatibles con el mercado interior "las ayudas [...] destinadas a poner remedio a una grave perturbación en la economía de un Estado miembro". Esta disposición, ampliamente utilizada durante la crisis económica de 2008 para proveer de asistencia financiera a las entidades de crédito, deja a la Comisión un mayor margen de discrecionalidad que la anterior a la hora de autorizar determinadas ayudas estatales (Santa María, Gambaro, Missanelli, 2020). Además, la comunicación avanzaba también la posibilidad de que otros Estados miembros se acogieran igualmente a este precepto, así como la elaboración de un marco jurídico especial con arreglo al mismo que no tardaría en ser adoptado. 
En efecto, por medio de una nueva comunicación de 19 de marzo, la Comisión aprobó un "marco temporal relativo a las medidas de ayuda estatal destinadas a respaldar la economía en el contexto del actual brote de covid-19"2. Aquí se autorizan cinco tipos de ayudas de Estado:

i) subvenciones directas, ventajas fiscales selectivas y anticipos por un importe máximo de 800.000 euros por empresa; ii) garantías estatales subvencionadas sobre los préstamos bancarios; iii) préstamos públicos con tipos de interés subvencionados; iv) ayudas en forma de garantías y préstamos canalizados a través de entidades de crédito $u$ otras entidades financieras (especificándose que se trata de ayudas directas a los clientes de los bancos y no a los propios bancos); v) seguro de crédito a la exportación a corto plazo.

La vigencia temporal de este marco jurídico se fija inicialmente hasta finales de diciembre de 2020, pudiendo ser prorrogado por la Comisión. Además, posteriormente ha sido ampliado con nuevas medidas al objeto de que los Estados miembros puedan acelerar la investigación, los ensayos y la producción de productos relacionados con el coronavirus.

Pese a que, en principio, el objetivo de este marco jurídico temporal consistía en permitir que los Estados miembros pudieran hacer frente a las dificultades financieras con que se fueran encontrando las empresas, esto debía lograrse manteniendo la integridad del mercado interior de la UE y garantizando la igualdad de condiciones. Sin embargo, lo cierto es que el muy diverso margen fiscal de los Estados miembros ha dado lugar a inmensas disparidades en el monto de las ayudas concedidas ${ }^{3}$, lo que con toda probabilidad tendrá un enorme impacto sobre el mercado interior una vez superada la crisis.

\footnotetext{
${ }^{2}$ Comisión Europea, "Marco Temporal relativo a las medidas de ayuda estatal destinadas a respaldar la economía en el contexto del actual brote de COVID-19" COM (2020) 1863 final, de 19 de marzo (DOUE C 91 del 20 de marzo de 2020, p.1). Este marco temporal fue posteriormente modificado mediante las comunicaciones C (2020) 2215, de 3 de abril de 2020 , C (2020) 3156, de 8 de mayo de 2020, y C(2020) 4509, de 29 de junio de 2020.

${ }^{3}$ Aunque en el momento en que se elabora este trabajo aún siguen aprobándose ayudas estatales por parte de la Comisión conforme al marco temporal analizado, a finales de junio de 2020 Alemania acaparaba entre el 44 y el $50 \%$ del total de ayudas autorizadas, muy por delante de Italia (19\%), Francia (17\%) y, desde luego, España (4\%). Alemania se lo lleva todo: España sigue a la cola europea en ayudas a empresas, La Información, disponible en: https://www.lainformacion.com/economia-negocios-y-finanzas/alemania-se-lleva-todo-espanasigue-cola-europea-ayudas-empresas/2808681/
} 
2. La flexibilización del marco presupuestario europeo

Como es lógico, la puesta en práctica de las ayudas de Estado a que acabamos de referirnos presupone su financiación, lo que ha obligado a los países miembros de la UE a obtener rápidamente enormes cantidades de recursos en los mercados financieros. No obstante, en la mayor parte de los casos esto no hubiera sido posible de haber permanecido inalterado el marco presupuestario europeo. Y es que, a grandes rasgos, el Pacto de Estabilidad y Crecimiento (PEC), en conjunción con el Tratado de Estabilidad, Coordinación y Gobernanza (TECG), imponen a sus Estados parte las obligaciones de no sobrepasar un límite de endeudamiento del 60\%, así como mantener un déficit estructural inferior al 0,5\%, ambos en relación con el PIB.

Pues bien, al objeto de permitir que la respuesta de los Estados a las consecuencias económicas ocasionadas por la pandemia no se viera constreñida por estas limitaciones, el 20 de marzo la Comisión Europea solicitó formalmente al Consejo la activación — por vez primera- de la denominada cláusula general de salvaguardia del $\mathrm{PEC}^{4}$.

Esta cláusula, introducida como parte de la reforma del PEC llevada a cabo en 2011, permite que los Estados miembros adopten medidas presupuestarias para hacer frente a situaciones de crisis generalizada causadas por una ralentización económica grave en la zona del euro o en el conjunto de la UE. Es decir, habilita a los Estados miembros para que, temporalmente y de forma coordinada y ordenada, puedan desviarse de los requisitos en materia de estabilidad presupuestaria dentro de los procedimientos preventivo y corrector del PEC.

Por lo que respecta al componente preventivo, el artículo 5, apartado 1, y el artículo 9, apartado 1, del Reglamento (CE) 1466/97 establecen que "[...] en períodos de crisis económica grave en la zona del euro o en el conjunto de la Unión, se podrá permitir a los Estados miembros que se aparten temporalmente de la trayectoria de ajuste hacia el objetivo presupuestario a medio plazo [...], siempre que dicha desviación no ponga en peligro la sostenibilidad presupuestaria a medio plazo". Por lo que se refiere al componente corrector, el artículo 3, apartado 5, y el artículo 5, apartado 2, del

\footnotetext{
${ }^{4}$ Comisión Europea, "Comunicación de la Comisión al Consejo relativa a la activación de la cláusula general de salvaguardia del Pacto de Estabilidad y Crecimiento", COM (2020) 123 final, de 20 de marzo.
} 
Reglamento (CE) 1467/97 disponen que, en esas mismas circunstancias excepcionales, el Consejo puede también decidir, previa recomendación de la Comisión, la adopción de una trayectoria presupuestaria revisada.

Tras la citada comunicación, el 23 de marzo el Consejo "EcoFin" (compuesto por los ministros de Hacienda de los Estados miembros), emitió una declaración ${ }^{5}$ en la que manifestaba su conformidad con la evaluación de la Comisión, afirmando que concurrirían las condiciones excepcionales previstas para la activación de la cláusula general de salvaguardia del marco presupuestario de la UE. De acuerdo con esta declaración, "la activación de la cláusula garantizará la flexibilidad necesaria para tomar todas las medidas que se requieran a fin de reforzar nuestros sistemas sanitarios y de protección civil y proteger nuestras economías, en particular mediante un estímulo adicional de carácter discrecional y una acción coordinada, concebida por los Estados miembros como consideren, que sea oportuna, temporal y específica".

\section{LA INTERVENCIÓN DEL BANCO CENTRAL EUROPEO}

Como se ha dicho, la flexibilización de los marcos normativos europeos que acaba de exponerse ha permitido el despliegue de políticas fiscales expansivas por parte de los Estados miembros para hacer frente a las consecuencias económicas ocasionadas por la pandemia. Un cuantioso incremento del gasto público que, lógicamente, ha supuesto un mayor endeudamiento estatal. No obstante, este endeudamiento se ha producido en condiciones diversas y ha tenido consecuencias distintas en función de la situación fiscal de los países emisores, que además se han visto afectados de forma asimétrica por el impacto del coronavirus ${ }^{6}$.

En efecto, la pandemia golpeó en primer lugar y de forma especialmente intensa a Italia, lo que pronto se tradujo en que el diferencial (spread) de su deuda pública — la denominada prima de riesgo - se disparase. Una circunstancia que, pese a ciertas dudas iniciales, propició una rápida respuesta por parte del Banco Central Europeo (BCE) al objeto de evitar una situación

\footnotetext{
${ }^{5}$ Consejo de la Unión Europea, comunicado de prensa 173/20, de 23 de marzo. Disponible en: https://www.consilium.europa.eu/es/press/press-releases/2020/03/23/statement-of-eu-ministersof-finance-on-the-stability-and-growth-pact-in-light-of-the-covid-19-crisis/pdf

${ }^{6}$ En este sentido, ya se ha mencionado el muy diverso margen de maniobra fiscal operado por las principales economías de la zona euro en forma de ayudas de Estado. Vid. Supra, nota 3.
} 
similar a la del verano de 2012. Así, tras un anuncio el día 12 de marzo por el que se incrementaba de forma temporal en 120.000 millones de euros el programa ampliado de adquisiciones de activos (PAA) ${ }^{7}$-insuficiente para calmar a los mercados-, el 18 de marzo se anunció un programa temporal de compras de emergencia en caso de pandemia (PEPP, por sus siglas en inglés) mucho más ambicioso ${ }^{8}$.

EI PEEP abarcará la compra por el BCE de todas las categorías de activos cuya adquisición venía siendo admisible al amparo del PAA, pero sus compras "serán independientes y adicionales a las realizadas de conformidad con el PAA, con una dotación global de 750.000 millones de euros hasta finales de 2020"9. Además, "el PEPP no hace referencia a las condiciones, por lo que renuncia de manera explícita a los requerimientos que se establecieron previamente para la compra de la deuda griega bajo la flexibilización cuantitativa" (Bobic y Dawson, 2020). A lo que hay que añadir que las compras de activos bajo este programa excepcional se efectuarán de forma flexible, sin necesidad de atender a la participación de los Estados de la Eurozona en el capital del BCE (el denominado capital key) ${ }^{10}$.

EI PEPP se enmarca en la senda de los programas Quantitative Easing abierta por el BCE durante la última crisis financiera de la zona euro. Como señalan Dani y Menéndez estos programas están en tensión, si no con el tenor, al menos sí con el espíritu del artículo 123 TFUE, precepto que prohíbe la adquisición directa de instrumentos de deuda a los Estados miembros por parte del BCE (Dani y Menéndez, 2020: 532). Además, pese a haber sido respaldados por el TJUE bajo determinadas condiciones, la reciente y polémica Sentencia del Tribunal Constitucional Federal Alemán de 5 de mayo de $2020^{11}$

\footnotetext{
${ }^{7}$ Banco Central Europeo, nota de prensa. Disponible en: https://www.bde.es/f/webbde/GAP/Secciones/SalaPrensa/ComunicadosBCE/DecisionesPolitica Monetaria/20/pm200312.pdf.

Además, al objeto de facilitar la disposición de liquidez se toman otras decisiones como la relajación temporal de los requerimientos de capital a las entidades de crédito:

https://www.bde.es/f/webbde/GAP/Secciones/SalaPrensa/ComunicadosBCE/DecisionesPolitica Monetaria/20/presbce2020_45.pdf

${ }^{8}$ Decisión (UE) 2020/440 del Banco Central Europeo, de 24 de marzo de 2020, sobre un programa temporal de compras de emergencia en caso de pandemia (BCE/2020/17). DOUE L 91, 1-4.

9 Ídem., Considerando 3.

${ }^{10}$ Decisión (UE) 2020/440 del BCE, artículos 5.1 y 5.2 .

${ }^{11}$ Sentencia del Tribunal Constitucional Federal Alemán de 5 de mayo de 2020, Segundo Senado, 2 BvR 859/15.
} 
ha puesto de manifiesto el precario soporte jurídico sobre el que operan estos programas (Dani y Menéndez, 2020: 533).

\section{LOS MECANISMOS FINANCIEROS DE LA UNIÓN EUROPEA PARA COMBATIR LAS CONSECUENCIAS ECONÓMICAS DE LA PANDEMIA}

1. El Instrumento europeo de apoyo temporal para mitigar los riesgos de desempleo en una emergencia

Una de las primeras y más evidentes consecuencias económicas ocasionadas por la pandemia de coronavirus ha sido su impacto sobre el empleo. En efecto, el confinamiento decretado en la práctica totalidad de los Estados miembros como medida para mitigar la propagación del virus se tradujo inmediatamente en el cierre de todas las empresas no esenciales. Para tratar de evitar que este parón en la actividad económica se tradujera en una destrucción masiva de empleo, los Estados, además de alentar el teletrabajo en la medida de lo posible, recurrieron a diversos expedientes para propiciar que las empresas se decantasen por la suspensión temporal de los contratos de trabajo en lugar de por su rescisión. Este ha sido el caso de los Kurzarbeitergeld en Alemania, la Cassa Integrazione Guadagni en Italia, o los ERTE en España.

Pues bien, de cara a facilitar que los Estados miembros más severamente golpeados por la pandemia pudieran financiar estos mecanismos, la Comisión Europea —que en su citada comunicación de 13 de marzo ya había anunciado medidas para aliviar las repercusiones sobre el empleo- presentó el 2 de abril una propuesta de Reglamento para crear el denominado "Instrumento europeo de apoyo temporal para mitigar los riesgos de desempleo en una emergencia"12 (SURE, por sus siglas en inglés).

El funcionamiento de este mecanismo es simple: se trata de un fondo dotado con hasta 100.000 millones de euros al que los Estados miembros podrán acudir para obtener financiación en forma de préstamos en condiciones favorables. El programa se financiará mediante emisiones de deuda de la Unión Europea en el mercado y, para evitar que el pasivo contingente derivado de esos préstamos de la Unión resulte incompatible con las restricciones del

${ }^{12}$ Comisión Europea, "Propuesta de Reglamento del Consejo relativo a la creación de un Instrumento Europeo de Apoyo Temporal para Mitigar los Riesgos de Desempleo en una Emergencia (SURE) a raíz del brote de COVID-19", COM (2020) 139 final, de 2 de abril. 
presupuesto de la UE, este se respaldará mediante un sistema de garantías de los Estados miembros al presupuesto de la Unión por valor del $25 \%$ de los préstamos que se concedan.

El 3 de agosto, el Ministerio de Economía informó mediante una nota de prensa $^{13}$ de que el Gobierno de España había solicitado el acceso a este nuevo mecanismo, presentando una propuesta de financiación superior a los 20.000 millones de euros destinada a cubrir el coste de los ERTE y de la prestación para autónomos. El importe final a percibir será determinado por parte del Consejo de la UE, previa propuesta de la Comisión, y dependerá, entre otros factores, de las cuantías que soliciten el resto de países interesados en el programa $^{14}$.

2. Medidas para facilitar la liquidez a través del Grupo del Banco Europeo de Inversiones

También en la comunicación de 13 de marzo sobre la "respuesta económica coordinada al brote de covid-19", la Comisión anunció ya la movilización de 1.000 millones de euros del presupuesto de la UE como garantía del Fondo Europeo de Inversiones (FEl) para apoyar la financiación de aproximadamente 8.000 millones de euros de capital circulante para las pymes y empresas de mediana capitalización en toda Europa. Una medida que, finalmente, fue lanzada por este organismo integrante del Grupo del Banco Europeo de Inversiones (BEI) el día 6 de abril. Además, el 16 de marzo el Grupo del BEI propuso un plan para movilizar hasta 40.000 millones de euros de financiación con la intención de destinarlos a préstamos puente, periodos de carencia y otras medidas destinadas a aliviar la falta de liquidez y de capital circulante de pequeñas y medianas empresas.

No obstante, la medida más destacable de las promovidas por el BEI es la creación de un fondo de garantía paneuropeo para hacer frente a las consecuencias económicas de la pandemia de covid-19. Este fondo, aprobado por el Consejo Europeo el 23 de abril y cuya estructura y modelo de negocio

\footnotetext{
13 Ministerio de Economía del Gobierno de España, nota de prensa. Disponible en: https://www.mineco.gob.es/stfls/mineco/prensa/noticias/2020/200803_np_SURE.pdf

${ }^{14}$ El 24 de agosto la Comisión Europea emitió un comunicado de prensa con sus propuestas de ayudas financieras con cargo al SURE. El monto total propuesto ascendía a 81.400 millones de euros, de los que 21.300 serían para España. Disponible en: https://ec.europa.eu/commission/presscorner/detail/es/IP_20_1496
} 
fueron decididos por el consejo de administración del BEI el 26 de mayo, está dotado con 25.000 millones de euros aportados por los 27 Estados miembros de la UE en proporción a su participación en el capital de la entidad. Esto permitirá al BEI movilizar hasta 200.000 millones de euros de financiación adicional para empresas — fundamentalmente pymes - viables a largo plazo pero que hayan experimentado problemas de liquidez a causa de la pandemia.

3. De la apuesta fallida por los "coronabonos" a la eliminación de la condicionalidad del MEDE

Las inmensas necesidades de financiación de los Estados de la UE a causa del descomunal incremento del gasto público para hacer frente a las consecuencias socioeconómicas de la pandemia pronto reabrieron un debate que parecía haber quedado zanjado tras la crisis financiera de 2008: la posible mutualización de deuda pública entre Estados de la Eurozona.

En efecto, durante la crisis de deuda soberana de 2010-2012, cuando la prima riesgo de algunos países de la Eurozona se disparó hasta niveles alarmantes, Francia e Italia propusieron emitir "eurobonos"; es decir, deuda mutualizada, prestada colectivamente por todos los países de la zona euro al amparo del Banco Central Europeo. Una propuesta basada en la idea de que esto permitiría que los Estados de la zona euro pudieran acceder en mejores condiciones a los mercados de capitales, ya que la responsabilidad del pago de esta deuda sería mancomunada. No obstante, lo cierto es que Alemania rechazó esta posibilidad de forma tajante y los "eurobonos" no llegaron a prosperar.

Pues bien, amparándose en el argumento de que la pandemia constituye un "impacto externo simétrico, del cual ningún país es responsable, pero cuyas consecuencias negativas nos afectan a todos", los Jefes de Estado o de Gobierno de nueve países de la UE -España, Italia, Francia, Bélgica, Luxemburgo, Irlanda, Portugal, Grecia y Eslovenia- solicitaron por carta al Presidente del Consejo Europeo "trabajar en un instrumento de deuda común emitida por una institución europea para obtener fondos en el mercado sobre la misma base y en beneficio de todos los Estados miembros"15.

15 La misiva, fechada el 25 de marzo de 2020, puede consultarse aquí: https://www.lamoncloa.gob.es/presidente/actividades/Documents/2020/25032020CartaDeLider es(ES).pdf 
Pese a las inmensas diferencias entre ambas crisis, esta propuesta volvió a encontrarse con la negativa de Alemania y, sobre todo, de los Países Bajos, cuyo Primer Ministro terminó por erigirse en adalid de un grupo de países radicalmente contrarios a la idea de emitir eurobonos, coloquialmente conocidos ahora como "coronabonos"16. Así, la propuesta de emisión de deuda mutualizada fue rechazada durante una tensa reunión del Consejo Europeo celebrada por videoconferencia el 26 de marzo, que en este punto se limitó a requerir del Eurogrupo una nueva propuesta de medidas en el plazo de dos semanas $^{17}$.

En cumplimiento de tal mandato, el 9 de abril los Ministros de finanzas de la zona euro presentaron una propuesta basada en tres "redes de seguridad europeas" para afrontar el embate socioeconómico de la pandemia: una para los trabajadores, otra para las empresas y una tercera para la deuda soberana. Las dos primeras cristalizarían finalmente en el SURE y el fondo de garantía paneuropeo del BEl, ya analizados. Por su parte - y como cabía esperar a raíz de las enfrentadas posturas del referido Consejo Europeo-, la safety net propuesta por el Eurogrupo para facilitar la financiación del gasto público estatal no estuvo basada en la mutualización de la deuda, sino en un conocido mecanismo intergubernamental de ingrato recuerdo en el sur de Europa: el Mecanismo Europeo de Estabilidad (MEDE).

EI MEDE fue el instrumento de asistencia financiera utilizado durante la última crisis para canalizar los denominados "rescates" a Grecia, Irlanda, Portugal y el sistema bancario español. Un mecanismo creado en 2012 mediante un tratado internacional suscrito por los Estados de la Eurozona y al margen del Derecho europeo que, sin embargo, atribuye importantes competencias a la Comisión Europea $^{18}$. En particular, a la hora de determinar y supervisar (junto con el BCE y el FMI, la denominada Troika) las condiciones de las líneas de crédito que los países "rescatados" debían asumir para poder acceder una financiación a intereses más favorables que los de mercado, especialmente en un contexto de primas de riesgo desbocadas. Esta condicionalidad se establecía en los

${ }^{16}$ Este grupo de países, integrado por Austria, Dinamarca y Suecia, además de por Países Bajos, pasaron a ser referidos por los medios de comunicación y la opinión pública como "países frugales".

17 La declaración conjunta de los miembros del Consejo Europeo de 26 de marzo puede consultarse aquí: https://www.consilium.europa.eu/media/43098/26-vc-euco-statement-es.pdf

${ }^{18}$ Sobre la creación del MEDE y el intento de dotar a este mecanismo de cobertura jurídica en el Derecho originario de la UE a través de la reforma del artículo 136 TFUE, vid. (MORENO, 2019: 251-258). 
denominados Memoranda of Understanding (MoU), y estaba "presidida en todo momento por la exigencia de consolidación fiscal, estabilidad presupuestaria y sostenibilidad financiera" (Moreno, 2019: 284), lo que en la práctica se traducía en drásticas reducciones del gasto público: los temidos "recortes".

Con estos antecedentes, no puede sorprender la resistencia que los países partidarios de la emisión de deuda mutualizada -y muy especialmente España e Italia - opusieron a la propuesta de reavivar el uso de este instrumento. No obstante, tras arduas negociaciones en el seno del Eurogrupo consiguió alcanzarse un acuerdo para habilitar una línea de crédito dentro del MEDE, pero exenta de condicionalidad. El único requisito para acceder a ella sería el de destinar los fondos recibidos a financiar el gasto sanitario directo o indirecto ocasionado por la pandemia. Así, el acuerdo de 9 de abril ${ }^{19}$ refleja una propuesta de línea de financiación denominada Pandemic Crisis Support y

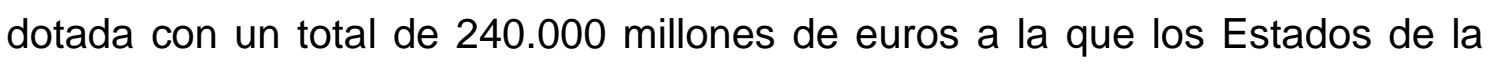
Eurozona podrían acceder para solicitar en préstamo hasta el $2 \%$ de su PIB. Esta propuesta - junto con el SURE y el fondo de garantía paneuropeo del $\mathrm{BEl}$ - fue aprobada por el Consejo Europeo de 23 de abril $^{20} \mathrm{y}$, tras ser ultimados sus detalles por el Eurogrupo el 8 de mayo ${ }^{21}$, el Consejo de Gobernadores del MEDE la hizo operativa el 15 de mayo.

Pese a todo, resulta sorprendente que, cinco meses después de estar disponible, en el momento en que se ha elaborado este trabajo, los países que tan esforzadamente negociaron para eliminar la condicionalidad de este mecanismo aún no hayan dado el paso de acogerse a él. $Y$ ni siquiera es seguro que vayan a hacerlo. La explicación de esta actitud seguramente resida en el estigma que aún hoy pesa sobre el MEDE, lo que dificulta que los Gobiernos recurran a este instrumento por el enorme coste político que se presupone a esta decisión ${ }^{22}$. Ahora bien, pese a que el mayor atractivo del

19 Conclusiones del Eurogrupo de 9 de abril de 2020, aptdo. 16. Disponible en: https://www.consilium.europa.eu/media/43373/20200407-eg-inclusive-summing-up-letter.pdf

${ }^{20}$ Conclusiones del Presidente del Consejo Europeo tras la videoconferencia celebrada por los Jefes de Estado y de Gobierno de la UE el 23 de abril de 2020. Nota de prensa de 23 de abril de 2020, 251/20. Disponible en: https://www.consilium.europa.eu/en/press/pressreleases/2020/04/23/conclusions-by-president-charles-michel-following-the-video-conferencewith-members-of-the-european-council-on-23-april-2020/pdf

21 Term sheet: ESM Pandemic Crisis Support. Disponible en: https://www.consilium.europa.eu/media/44011/20200508-pcs-term-sheet-final.pdf

${ }^{22}$ Hasta tal punto ha llegado esta cerrazón que el propio MEDE publicó el 29 de julio una entrada en su blog explicando las virtudes de esta línea de crédito en términos de ahorro para 
Pandemic Crisis Support resida en que sus fondos están disponibles inmediatamente, en la decisión de no acogerse a esta línea de crédito probablemente influya también la existencia de un enorme plan de recuperación cuya negociación ya se vislumbraba en el momento en que se aprobaron las tres "safety nets" que acabamos de exponer. Un plan que terminaría por cristalizar en un fondo de recuperación sin precedentes en la historia de la UE y cuya concepción y pormenores trato de explicar a continuación.

\section{LA GESTACIÓN DEL PLAN DE RECUPERACIÓN EUROPEO FRENTE A LA PANDEMIA: NEXT GENERATION EU}

Como acaba de apuntarse, en las conclusiones del Eurogrupo de 9 de abril ya se anunciaba la negociación de un fondo to prepare and support the recovery, providing funding through the EU budget to programmes designed to kick-start the economy in line with European priorities and ensuring EU solidarity with the most affected member states ${ }^{23}$.

Conviene recordar que, al fin y al cabo, de acuerdo con el artículo 3.3 TUE, "Ia Unión fomentará la cohesión económica, social y territorial y la solidaridad entre los Estados miembros". Sin embargo, en el momento de emitirse las citadas conclusiones, los Ministros de finanzas de la zona euro albergaban concepciones muy dispares sobre cómo habría de financiarse y articularse dicho fondo. Así, volvió a hacerse patente la división entre países del norte y del sur de Europa. Los primeros, liderados por Alemania, Países Bajos y Austria, abogaban por que este mecanismo de recuperación estuviera basado en préstamos a los países más afectados por la pandemia. Por su parte, los países del sur, abanderados por Francia, España e Italia, apostaban porque el fondo se repartiera mediante subsidios no reembolsables y porque estos se financiasen a través de la emisión de deuda mutualizada. Como señala Utrilla, the issue is a crucial one for the sustainability of the EU itself, since grants presuppose increased solidarity by the less affected countries, whilst loans would increase the debts of Member States with economies already weak

los Estados que se acogieran a ella. ANEV JANSE, K. y RUHL, S.: Why the Covid-19 credit line still makes sense. Disponible en: https://www.esm.europa.eu/blog/why-covid-19-credit-line-stillmakes-sense

${ }^{23}$ Conclusiones del Eurogrupo de 9 de abril de 2020, aptdo. 19. Vid., supra, n. 19. 
before the pandemic, thereby widening the gap between the strongest and weakest economies in the common market (Utrilla, 2020a).

Las referidas posiciones de los Estados miembros parecían irreconciliables hasta que, sorpresivamente, el 18 de mayo Alemania manifestó un viraje radical en su postura ${ }^{24}$. En efecto, en una declaración conjunta de la Canciller alemana Angela Merkel y del Presidente francés Emmanuele Macron, se anunció una propuesta de ambos países para la creación de un fondo de recuperación europeo dotado con 500.000 millones de euros. Un montante que, según ambos mandatarios, habría de repartirse en forma de subsidios entre las regiones de Europa y los sectores económicos más afectados por la pandemia. Además, para financiar este plan de recuperación excepcional se proponía autorizar a la Comisión Europea para que emitiese obligaciones en los mercados de capitales en nombre de la $U E^{25}$.

El mismo día 18 de mayo, la Presidenta de la Comisión emitió un comunicado ${ }^{26}$ en el que agradecía la propuesta franco-alemana y afirmaba que ésta iba en la misma línea que el plan de recuperación en el que venía trabajando el Ejecutivo comunitario. Un plan que pocos días antes había sido presentado ante el Parlamento Europeo ${ }^{27}$.

\section{La propuesta de la Comisión Europea}

Pues bien, poco más de una semana después, el 27 de mayo, la Comisión Europea hizo su propia propuesta para un plan de recuperación ${ }^{28}$. Una propuesta que descasaba esencialmente sobre dos pilares: un fondo extraordinario de recuperación denominado Next Generation EU y un Marco

\footnotetext{
${ }^{24}$ Sobre el cambio de postura de Alemania resulta esclarecedora la siguiente pieza del Financial Times de 9 de junio de 2020: The minds behind Germany's shifting fiscal stance, disponible en: https://www.ft.com/content/2503ce9c-cde9-4301-bba0-8301f7deaf3b

${ }^{25}$ Los detalles de la propuesta franco-alemana pueden consultarse en la siguiente nota de prensa del Gobierno Federal alemán:

https://www.bundeskanzlerin.de/resource/blob/656734/1753772/414a4b5a1ca91d4f7146eeb2b 39ee72b/2020-05-18-deutsch-franzoesischer-erklaerung-eng-data.pdf

${ }^{26}$ Comisión Europea, comunicado de prensa. Disponible en: https://ec.europa.eu/commission/presscorner/detail/en/statement_20_902

27 Discurso de la Presidenta de la Comisión Europea, Ursula von der Leyen ante el ante el Pleno del Parlamento Europeo el 13 de mayo de 2020. Disponible en: https://ec.europa.eu/commission/presscorner/detail/en/speech_20_877

${ }^{28}$ Comisión Europea, "El momento de Europa: reparar los daños y preparar el futuro para la próxima generación", $\operatorname{COM}(2020) 456$ final, de 27 de mayo.
} 
Financiero Plurianual (MFP) revisado para el periodo 2021-2027 al que aquel estaría vinculado en gran medida.

Por lo que se refiere al Next Generation EU, la composición del fondo sería semejante a la propuesta por Francia y Alemania, aunque sería todavía más ambicioso. Así, estaría integrado por 500.000 millones de euros que se pondrían a disposición de los Estados miembros a través de subvenciones; pero, además, a estos se sumarían otros 250.000 millones que serían transferidos en forma de préstamos.

El total de esos 750.000 millones se recaudarían gracias a la emisión de obligaciones en los mercados financieros y, para poder hacerlo, la Comisión propuso elevar "temporalmente" el límite máximo de recursos propios al $2 \%$ de la renta nacional bruta de la UE. Una vez recaudados, estos fondos adicionales serían canalizados a través de distintos programas y devueltos con cargo a los futuros presupuestos de la UE a partir de 2028 y hasta 2058 a más tardar.

Con vistas a financiar la devolución de los pasivos generados por este fondo excepcional de recuperación, la comunicación afirmaba que la Comisión propondría una serie de nuevos recursos propios. Entre ellos se menciona uno basado en el régimen de comercio de derechos de emisión, un mecanismo de ajuste en frontera de las emisiones de carbono y un recurso propio basado en las actividades de las grandes empresas. Además, la propuesta abre la puerta a imponer una tasa digital, a lo que habría que añadir los planes de la Comisión relativos a un impuesto sobre el valor añadido simplificado y sobre los plásticos no reciclados.

En cuanto a la inversión de los fondos recaudados, estos se destinarían por medio de diversos programas a tres cuestiones fundamentales: apoyar a los Estados miembros en sus inversiones y reformas para hacer frente a la crisis, relanzar la economía de la UE incentivando la inversión privada, y reforzar de la actuación estratégica de la UE en materia sanitaria y exterior.

Por otro lado, la Comisión propuso también un marco financiero plurianual revisado para el periodo 2021-2027 dotado con 1,1 billones de euros ${ }^{29}$. Un presupuesto a largo plazo que iría invirtiéndose en los mismos tres pilares que el fondo de recuperación extraordinario y que tendría como objetivo prioritario potenciar la doble transición ecológica y digital de la Unión Europea. Además,

${ }^{29}$ Comisión Europea, "El presupuesto de la UE: motor del plan de recuperación para Europa". $\operatorname{COM}(2020) 442$ final, de 27 de mayo. 
con el fin de poner fondos a disposición de los Estados miembros cuanto antes, la Comisión propuso modificar el marco financiero plurianual 2014-2020 para posibilitar una financiación adicional de 11.500 millones de euros ya en 2020 .

\section{La negociación del plan de recuperación en el Consejo Europeo}

Como era de esperar, en torno a la propuesta de la Comisión sobre los mecanismos de recuperación volvieron alinearse dos bloques enfrentados de Estados miembros. Por un lado, los denominados cuatro frugales (Países Bajos, Austria, Dinamarca y Suecia) que, junto con Finlandia, se opusieron tanto a la cuantía del fondo de recuperación excepcional como al sistema de subvenciones no reembolsables, siendo partidarios de un mecanismo basado en préstamos en condiciones favorables. Por otro lado, el resto de Estados miembros, partidarios de sacar adelante la propuesta de la Comisión y, de nuevo, capitaneados por Francia, España e Italia, aunque ahora también con el importante respaldo de Alemania.

Estas discrepancias se pusieron de manifiesto en la reunión del Consejo Europeo celebrada por videoconferencia el 19 de junio de 2020 y, a partir de entonces, su Presidente celebró múltiples reuniones bilaterales para tratar de acercar las diversas posturas. Como resultado, el 10 de julio el Presidente del Consejo Europeo, Charles Michel, presentó su propuesta para el fondo de recuperación y el MFP ${ }^{30}$. Una propuesta que, si bien partía de la efectuada por la Comisión, introducía algunos cambios importantes sobre sus puntos más controvertidos.

Así, se reducía la cuantía propuesta para el MFP a 1,074 billones de euros. Se apostaba por mantener el sistema de correcciones para los países frugales y Alemania; es decir, la posibilidad de reducir las contribuciones de estos países a los fondos de la UE. En cuanto al fondo de recuperación, se mantenía la autorización para que la Comisión emitiera deuda en los mercados financieros por valor de 750.000 millones de euros, pero se apostaba por una composición equilibrada - sin especificar- del uso de los fondos entre préstamos, garantías y subvenciones. Se establecían también una serie de condiciones para los Estados beneficiaros de los fondos, que tendrían que presentar a la

${ }^{30}$ Consejo Europeo, "El presidente Charles Michel presenta su propuesta para el MFP y el paquete de recuperación". Comunicado de prensa de 10 de julio de 2020. Disponible en: https://www.consilium.europa.eu/es/press/press-releases/2020/07/10/president-charles-michelpresents-his-proposal-for-the-mff-and-the-recovery-package/pdf 
Comisión planes nacionales de recuperación en consonancia con los objetivos del Semestre Europeo. Unos objetivos centrados, principalmente, en la transición ecológica y el respeto del Estado de Derecho. Sin embargo, se eliminaba la posibilidad de veto que habían reclamado los países frugales, sustituyéndola por un sistema de control de los planes nacionales de recuperación, que habrían de ser autorizados por mayoría cualificada del Consejo a propuesta de la Comisión.

Por último, la propuesta del Presidente del Consejo proponía adelantar el pago de los pasivos generados por el endeudamiento comunitario a 2026, lo que según él mismo agudizaría la presión para que la UE se dotase de nuevos recursos propios. En este sentido, se proponía a la Comisión Europea que diseñase tres nuevos recursos propios para ser introducidos a lo largo de 2021: uno sobre la utilización de residuos plásticos, otro consistente en una medida de ajuste de las emisiones de carbono y, por último, una tasa digital.

Finalmente, en el Consejo Europeo celebrado entre el 17 y el 21 de julio -el más largo de los últimos veinte años-, logró alcanzarse un acuerdo entre los Jefes de Estado y de Gobierno de la Unión Europea sobre el plan para la recuperación de los efectos económicos ocasionados por la pandemia de covid-19 ${ }^{31}$. En palabras de Utrilla, este acuerdo constitutes a historical landmark in the EU's budgetary and fiscal history, by opening the door for the first time ever to joint debt issuance and to (future) joint taxes (Utrilla, 2020b). Los puntos más relevantes de este acuerdo son los siguientes.

En primer lugar, la cuantía del fondo de recuperación Next Generation EU será finalmente de 750.000 millones de euros que, efectivamente, serán recaudados a través de la emisión de empréstitos por la Comisión Europea en los mercados de capitales en nombre de la $U^{32}$. Para habilitar jurídicamente esta posibilidad $-\mathrm{y}$ dado que será la primera vez que se emita deuda conjunta de esta forma - se procederá a reformar la Decisión sobre recursos propios de la $\mathrm{UE}^{33}$. Además, "los importes de los límites máximos de recursos propios se incrementarán temporalmente en 0,6 puntos porcentuales con el único fin de cubrir todos los pasivos de la Unión derivados de los empréstitos que haya

\footnotetext{
31 Conclusiones del Consejo Europeo del 17 al 21 de julio de 2020. Disponibles en: https://www.consilium.europa.eu/es/press/press-releases/2020/07/21/european-councilconclusions-17-21-july-2020/

${ }^{32}$ Ibid., aptdo. A5.

${ }^{33}$ Decisión del Consejo de 26 de mayo de 2014 sobre el sistema de recursos propios de la Unión Europea.
} 
contraído para hacer frente a las consecuencias de la crisis de la covid-19, hasta que todos estos pasivos se hayan extinguido, y a más tardar hasta el 31 de diciembre de $2058^{\prime 34}$.

Por lo que se refiere a la composición del fondo, este fue uno de los extremos donde más claramente se puso de manifiesto el compromiso alcanzado entre Estados miembros. En este sentido, solo 390.000 millones de euros serán desembolsados en forma de subvenciones no reembolsables para los países más afectados por el virus, mientras que los restantes 360.000 millones lo serán a través de préstamos ${ }^{35}$.

Los fondos serán distribuidos por medio de siete programas distintos, entre los que claramente destaca por volumen monetario el denominado "Mecanismo de Recuperación y Resiliencia" ${ }^{36}$. Al objeto de movilizar con rapidez la ayuda de este programa, el $70 \%$ de las subvenciones que lo integran se comprometerán en 2021 y 2022, mientras que el $30 \%$ restante se comprometerá enteramente a finales de $2023^{37}$.

Para poder acceder a los fondos de este mecanismo, "los Estados miembros elaborarán planes nacionales de recuperación y resiliencia que expongan el programa de reformas e inversiones del Estado miembro en cuestión para el periodo 2021-2023”38. Estos planes, que serán evaluados por la Comisión Europea en el plazo de dos meses tras su presentación, habrán de ser coherentes con las recomendaciones específicas para cada país y estar orientados hacia a la transición ecológica y digital. Finalmente, será el Consejo el que, "por mayoría cualificada y a propuesta de la Comisión, aprobará la evaluación de los planes de recuperación y resiliencia mediante un acto de ejecución que el Consejo procurará adoptar en un plazo de 4 semanas a partir de la propuesta. La evaluación positiva de las solicitudes de pagos estará supeditada al cumplimiento satisfactorio de las metas y los objetivos pertinentes" 39 . Por tanto, no se contempla la posibilidad de veto individual que

\footnotetext{
${ }^{34}$ Conclusiones del Consejo Europeo del 17 al 21 de julio de 2020, aptdo. A9.

35 Ibid., aptdo. A6.

${ }^{36}$ Este instrumento estará dotado con 672.500 millones de euros, de los que 360.000 millones se concederán en forma préstamos y 312.500 millones como subvenciones. Los otros seis mecanismos son "REACT-EU" (47.500 millones), "Horizonte Europa" (5.000 millones), "InvestEU" (5.600 millones), "Desarrollo rural" (7.500 millones), "Fondo de transición justa" (10.000 millones) y "resEU" (1.900 millones). Ibid., aptdo. A14.

${ }^{37}$ Conclusiones del Consejo Europeo del 17 al 21 de julio de 2020, aptdo. 15.

${ }^{38}$ Ibid., aptdo. A18.

${ }^{39}$ Ibid., aptdo. A19.
} 
propugnaban los países frugales. No obstante, estos sí que podrán supervisar el empleo de los fondos comunitarios pues, "en el caso excepcional de que uno o más Estados miembros consideren que existen desviaciones graves del cumplimiento satisfactorio de las metas y los objetivos pertinentes, podrán solicitar al presidente del Consejo Europeo que remita la cuestión al próximo Consejo Europeo" ${ }^{40}$. Y esto podría traducirse en un retraso en el desembolso de los fondos de hasta tres meses.

Pese a que, como acaba de apuntarse, los objetivos en materia ecológica ocuparán un lugar central en la evaluación de los planes de recuperación nacionales, la propuesta del Presidente del Consejo Europeo de que la disposición de los fondos estuviera también vinculada al respeto del Estado de Derecho finalmente no se refleja en las conclusiones del Consejo Europeo. Como señala Utrilla, the need to reach a unanimous agreement and the foreseeable impact of this point of the proposal on certain eastern European countries (especially Poland and Hungary) has diluted it in the final agreement, which merely mentions that "the European Council underlines the importance of the respect of the rule of law (Utrilla, 2020b).

Por otro lo referente al MFP 2021-2027, su dotación de acuerdo con las conclusiones del Consejo Europeo será de 1,0743 billones de euros y, por tanto, en línea con la ya mencionada propuesta de su Presidente. El acuerdo alcanzado confirma también la introducción de algunos de los nuevos recursos propios propuestos por la Comisión. En este sentido, se apuesta por un nuevo ingreso sobre los residuos plásticos no reciclados que se aplicará a partir del 1 de enero de 2021. De igual manera, se interpela a la Comisión para que, en el primer semestre de 2021, presente propuestas relativas a un mecanismo de ajuste en frontera de las emisiones de carbono y a un impuesto digital, con la intención de que ambos se instauren antes del 1 de enero de 2023. Además, se contempla también una propuesta de revisión del régimen de comercio de derechos de emisión que podría hacerse extensivo al transporte aéreo y marítimo; y se anuncia que "la Unión trabajará para instaurar otros recursos propios, entre los que podría hallarse un impuesto sobre las transacciones financieras" ${ }^{41}$.

Para finalizar, interesa destacar que las conclusiones del Consejo Europeo recogen también otra de las exigencias clásicas de los países frugales: la

\footnotetext{
${ }^{40}$ Ibidem.

${ }^{41}$ Conclusiones del Consejo Europeo del 17 al 21 de julio de 2020, aptdo. 29.
} 
reducción bruta de las contribuciones anuales de Dinamarca, Alemania, Países Bajos, Austria y Suecia para el periodo $2021-2027^{42}$.

\section{CONCLUSIONES}

Como se ha visto a lo largo de este trabajo, la respuesta de la Unión Europea a las consecuencias económicas ocasionadas por la pandemia de covid-19 ha sido radicalmente distinta a la que se articuló para hacer frente a la crisis financiera desencadenada en 2008. En este sentido, y pese a ciertas dudas iniciales, la UE ha activado todos los mecanismos de que disponía para facilitar que sus Estados miembros pudieran accionar cuantiosas políticas de gasto público, suspendiendo incluso la normativa europea sobre aspectos ya paradigmáticos del diseño jurídico comunitario como las ayudas de Estado y, sobre todo, el marco presupuestario.

Además, la propia UE ha desplegado novedosos mecanismos financieros de emergencia para respaldar la actuación de los Estados más golpeados por la pandemia. Así, incluso cuando ha habilitado mecanismos ya existentes como el MEDE lo ha hecho prescindiendo de la condicionalidad de sus préstamos, tratando con ello de eliminar uno de los aspectos más inclementes de cuantos se recuerdan en relación con la gestión de la última crisis.

Desde mi punto de vista, todas estas circunstancias propician que la solidaridad entre Estados —uno de los pilares básicos de la integración europea-, salga considerablemente reforzada en esta ocasión. Es cierto que tampoco ahora han prosperado propuestas como las relativas a la mutualización de la deuda. No obstante, la negociación de un fondo de recuperación sin precedentes en la historia de la UE — tanto por su cuantía como, sobre todo, por sus formas de financiación- abre la puerta a nuevos avances en la construcción comunitaria que hace tiempo se vienen reclamando, como los relativos a la integración fiscal.

\footnotetext{
42 Ibid., aptdo. 30.
} 
VII. BIBLIOGRAFÍA

- BOBIC, A. y DAWSON, M., "COVID-19" y el BCE: ¿serán las bases legales de la Unión Económica y Monetaria las próximas víctimas?", Agenda Pública, 3 de mayo de 2020. Disponible en: http://agendapublica.elpais.com/covid-19-y-elbce-seran-las-bases-legales-de-la-union-economica-y-monetaria-las-proximasvictimas/

- DANI, M. y MENÉNDEZ, A., "Le prime risposte dell'Unione Europea alle conseguenze economiche della crisi CoViD-19", BioLaw Journal - Rivista di BioDiritto, special issue 1/2020, pp. 527-539.

- MORENO GONZÁLEZ, G., Estabilidad presupuestaria y Constitución. Fundamentos teóricos y aplicación desde la UE, Tirant lo Blanch, Valencia, 2019.

- SANTA MARÍA, A., GAMBARO, E., y MISSANELLI, P.: "EU at the time of COVID-19", National Law Review, 5 de mayo de 2020. Disponible en: https://www.natlawreview.com/article/eu-time-covid-19

- UTRILLA, D., (a) "A way out of the crisis? The Franco-German proposal for a 500 billion-euro European Recovery Fund", EU Law Live, 19 de mayo de 2020. Disponible en: https://eulawlive.com/a-way-out-of-the-crisis-the-franco-germanproposal-for-a-500-billion-euro-european-recovery-fund/

- (b) "The European deal for post-pandemic economic recovery: content and meaning", EU Law Live, 21 de julio de 2020. Disponible en: https://eulawlive.com/the-european-deal-for-post-pandemic-economicrecovery-content-and-meaning/ 